\title{
Was war, was ist, was wird sein?
}

\section{Blick zurück nach vorn}

Wie - so fragt man sich häufig - soll es mit der Sozialen Arbeit eigentlich weitergehen? Wie vielen Unkalkulierbarkeiten wird dieses ohnehin so weitläufige und unübersichtlich angelegte Berufsfeld noch ausgesetzt sein und wie viel Drunter und Drüber wird es auch in Zukunft selbst erzeugen? Als da sind u.a.: die Unmengen unterschiedlichster Ausbildungsgänge im Spannungsfeld zwischen Universitäten und Fachhochschulen, ergänzt durch einen gigantischen Markt von Fort- und Weiterbildungsangeboten.

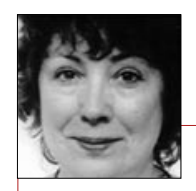

Sabine Hering *1947

Dr., Professorin an der Universität Siegen. hering@paedagogik. uni-siegen.de
Weiterhin finden wir dort die sagenumwobene Balance zwischen öffentlicher und privater Wohlfahrt, im Klartext: das nicht enden wollende Gerangel zwischen den unterschiedlich positionierten und privilegierten Trägern. Außerdem eine sich ständig wandelnde Klientel mit immer neuen Problemstrukturen, und, last, but not least: eine Anhäufung von Methoden und Konzepten, Theorien und Analysen, denen gegenüber der Turm zu Babel wie ein schlichtes Modell aus dem Lego-Katalog aussieht.

All dies ist keineswegs neu. Wir haben uns an die Unübersichtlichkeit gewöhnt, wir haben gelernt, mit der Komplexität und den Widersprüchen umzugehen. Zum Glück ist es uns sogar vergönnt, das gesamte Feld, auf das wir so häufig nachdenklich und gelegentlich sogar sorgenvoll blicken, insgesamt als ein durchaus erfolgreiches System wahrzunehmen ein System, das nicht selten viel besser funktioniert als manche der anderen, verwandten, aber sehr viel wohl geordneteren Welten.

Das hat vielerlei Gründe. Hans Falck hat mal gesagt: „Wenn man die Soziale Arbeit heute abschaffen würde, müsste sie morgen wieder erfunden werden. Unsere Gesellschaft braucht sie einfach." Und von Siegfried Mrochen stammt das Zitat: „Die Soziale Arbeit kann Probleme lösen, auf welche alle anderen Berufsgruppen (seien es Lehrer, Psychologen, Polizisten etc.) nur mit Hilflosigkeit reagieren."

Wie diese seltsame Erfolgsstory zu erklären ist und wer da an den richtigen Schrauben gedreht hat, ist eine Frage, die ganz sicherlich niemals schlüssig beantwortet werden kann. Wir haben uns aber trotzdem aufgemacht, diejenigen Schlüsselfiguren, von denen wir annehmen, dass sie in den vergangenen Jahrzehnten wesentliche Beiträge dazu geleistet haben, die Soziale Arbeit zu dem zu machen, was sie heute ist, zu ihrer Sicht auf die Vergangenheit, Gegenwart und Zukunft der Profession zu befragen.

Fast von allen, die wir zu dieser Form des ,Bekenntnisses' eingeladen haben, bekamen wir mehr oder weniger unverzüglich eine Zusage. Die ehemals führenden Protagonisten der Sozialen Arbeit sind also durchaus keine ,Oldies', die jetzt behaglich ihre Füße hochlegen und den lieben Gott einen guten Mann sein lassen, sondern es sind Persönlichkeiten, die nach wie vor sehr intensiv und kritisch die Entwicklungen dessen verfolgen, das sie selber mit in die Wege geleitet haben oder das sich - vice versa - ihren Plänen entgegengesetzt hat.Die Lektüre der folgenden Statements (von Rudolph Bauer, Lothar Böhnisch, Franz Hamburger, C. Wolfgang Müller und Hans Thiersch) belegt die Präzision der Beobachtungsgabe der Autoren und die Klarheit ihrer sozialpolitischen Ideen ebenso wie die bemerkenswerte persönliche Courage, die sie versuchen, den ,Nachgeborenen' mit auf den Weg zu geben. Ihre Aussagen sind wichtige Dokumente zum Verständnis der Probleme ebenso wie der Chancen, welche die Profession der Sozialen Arbeit gegenwärtig ausgesetzt ist. Sie repräsentieren keinen beliebigen ,Zeitgeist', sondern eine gut begründete und begehbare Brücke von der unmittelbaren Vergangenheit in die Zukunft.

Nur eines hat sich bei dieser Umfrage als problematisch erwiesen: Das war die Frage nach dem eigenen Beitrag, nach den persönlichen Verdiensten im Rahmen all der Fortschritte, welche die Soziale Arbeit in den vergangenen Jahren zu verzeichnen hatte. Diese Frage hat Reaktionen ausgelöst, welche als eindeutiger Beweis dessen dienen können, dass die Wissenschaft der Sozialen Arbeit ganz nachdrücklich geprägt ist von den Charakteristika, welche die Soziale Arbeit grundsätzlich auszeichnen: Viel zu viel Bescheidenheit, mangelnde Eitelkeit und der ganz und gar fehlende Hang zum Auftritt im Scheinwerferlicht. Das, finde ich, muss anders werden.

Abstract / Das Wichtigste in Kürze Fünf wichtige Persönlichkeiten der Sozialen Arbeit blicken auf ihre Arbeit und Wirkung zurück und versuchen außerdem eine Prognose zu künftigen Herausforderungen der Profession.

Keywords / Stichworte Einschätzung, Rückblick, Herausforderungen, Fortschritt, Zeitgeist, Zukunft. 